\title{
The Effects of Total Variation (TV) Technique for Noise Reduction in Radio-Magnetic X-ray Image: Quantitative Study
}

\author{
Kanghyen Seo ${ }^{1}$, Seung Hun Kim ${ }^{1}$, Seong Hyeon Kang ${ }^{1}$, Jongwoon Park ${ }^{1}$, \\ Chang Lae Lee ${ }^{2}$, and Youngjin Lee ${ }^{1 *}$ \\ ${ }^{1}$ Department of Radiological Science, Eulji University, 553, Sanseong-daero, Sujeong-gu, Seongnam-si, \\ Gyeonggi-do 13135, Korea \\ ${ }^{2}$ Department of Radiological Science, Yonsei University, 1, Yonseidae-gil, Wonju-si, Gangwon-do 26493, Korea
}

(Received 15 September 2016, Received in final form 13 December 2016, Accepted 15 December 2016)

\begin{abstract}
In order to reduce the amount of noise component in X-ray imaging system, various reduction techniques were frequently used in the field of diagnostic imaging. Although the previous techniques -such as median, Wiener filters and Anscombe noise reduction technique - were able to reduce the noise, the edge information was still damaged. In order to cope with this problem, total variation (TV) noise reduction technique has been developed and researched. The purpose of this study was to evaluate and compare the image quality using normalized noise power spectrum (NNPS) and contrast-to-noise ratio (CNR) through simulations and experiments with respect to the above-mentioned noise reduction techniques. As a result, not only lowest NNPS value but also highest CNR values were acquired using a TV noise reduction technique. In conclusion, the results demonstrated that TV noise reduction technique is proved as the most practical method to ensure accurate denoising in X-ray imaging system.
\end{abstract}

Keywords : radio-magnetic wave, X-ray radiography and digital radiography (DR), medical application, noise reduction technique, total variation

\section{Introduction}

With the advances in imaging techniques, $\mathrm{X}$-ray images based on general radiography and computed tomography (CT) were utilized as an essential method in clinical diagnostics. These images provide a lot of information about patients. However, the image noise still occurs because of the interaction between X-ray source and patients. X-ray images are usually suffered from this noise that restricts the diagnostic accuracy. The recent issue of X-ray images is the high amount of noise components. Noise reduction is the process of elimination of noise from an acquired signal. Appropriate filter and algorithm were required to reduce the noise in images. Although the median filter based on median value in the designated area was frequently used in the medical imaging field, different techniques for the noise reduction have been recently developed [1-3]. Among these techni-

CThe Korean Magnetics Society. All rights reserved.

*Corresponding author: Tel: +82-31-740-7264

Fax: +82-31-740-7264, e-mail: radioyoungj@gmail.com ques, Anscombe noise reduction technique and Wiener filter helped stabilization of noise variance such as PoissonGaussian noise. Markku M"akitalo et al. and Jingdong Chen et al. suggested effective Poisson-Gaussian noise reduction with improved Ascombe noise reduction technique or Wiener filter [4-6]. Especially, Anscombe noise reduction technique is useful in various inverse problems with Poisson noise contamination. As mentioned earlier, the noise reduction techniques are very effective for improving image performance; however, the edge with high frequency components is removed. Leonid I. Rudin et al. demonstrated that TV noise reduction technique can acquire sharp edge in the image [7-9]. However, a few of quantitative studies have been conducted with respect to the noise reduction techniques. The purpose of this study was to quantitatively evaluate and compare the image performances using various noise reduction techniques. For that purpose, we evaluated normalized noise power spectrum (NNPS) and contrast-to-noise ratio (CNR) with simulation and experiment.

The remainder of this paper is organized as follows: In section 2, the process of TV noise reduction technique 


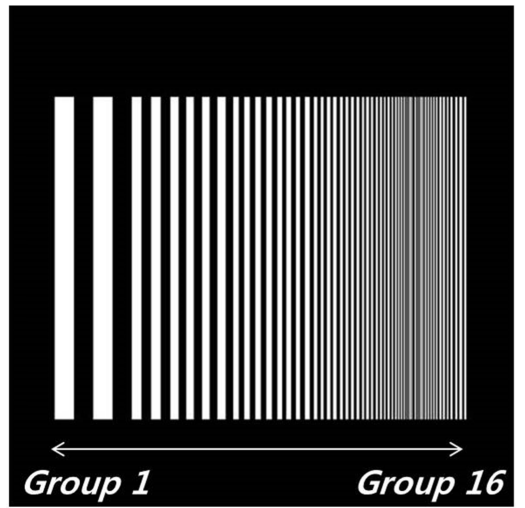

\begin{tabular}{c|c||c|c}
\hline \hline Group & $\begin{array}{c}\text { Spatial frequency } \\
(\mathrm{Ip} / \mathrm{mm})\end{array}$ & Group & $\begin{array}{c}\text { Spatial frequency } \\
(\mathrm{I} / \mathrm{mm})\end{array}$ \\
\hline 1 & 0.25 & 9 & 2.60 \\
2 & 0.50 & 10 & 3.47 \\
3 & 0.61 & 11 & 5.21 \\
4 & 0.87 & 12 & 10.42 \\
5 & 1.04 & 13 & 5.21 \\
6 & 1.49 & 14 & 3.47 \\
7 & 1.74 & 15 & 2.60 \\
8 & 2.08 & 16 & 2.08 \\
\hline \hline
\end{tabular}

Fig. 1. The photo of resolution phantom image with various spatial frequency groups for the simulation study.

using phantom is described. In section 3, NNPS and CNR using a region of interest (ROI) in the phantom are calculated to compare between TV and other noise reduction techniques. In section 4, the image performance with noise reduction techniques is analyzed and the usefulness of TV noise reduction technique is proved.

\section{Materials and Methods}

\subsection{Total variation (TV)}

Standard image degradation is often modeled as follows:

$$
g(x, y)=u(x, y)+n(x, y)
$$

where $u(x, y)$ is the desired clean image, $n(x, y)$ represents error (i.e., Gaussian noise), and the $g(x, y)$ is degraded image. To obtain the recovery image, many researchers have been studying the inverse problem such as spline smoothing, filtering using Fourier and wavelet transforms, and non-local self-similarity based methods [10-13]. However; in comparison to the least square methods where closed form, the linear solutions are well understood and easily computed. Also, the $1_{1}$-estimation is non-linear and computationally complex [14]. For that reason, we investigated for the denoising images by minimizing the TV norm of the estimated solution. Here, the TV can be written as:

$$
\begin{gathered}
\|u\|_{T V}=\sum_{i=1 j=1}^{M} \sum_{j=1}^{N}\left|\nabla u_{i, j}\right|=\sum_{i=1 j=1}^{M} \sum_{\left(u_{i, j}-u_{i-1, j}\right)^{2}+\left(u_{i, j}-u_{i-1, j}\right)^{2}} \\
u^{*}=\operatorname{argminTV}(\mathrm{u})
\end{gathered}
$$

where $M$ and $N$ are the numbers of rows and columns of the image $u$, respectively. Basically, TV from signals indicates the change of information from neighboring pixels in the image and the summation of all the difference in adjacent pixels' gradient. Then, iterative algorithms using the advantages of TV minimize each pixel gradient in the image.

\subsection{System geometry and phantom}

Figure 1 shows the used resolution phantom image in simulation which consisted of sixteen groups with different

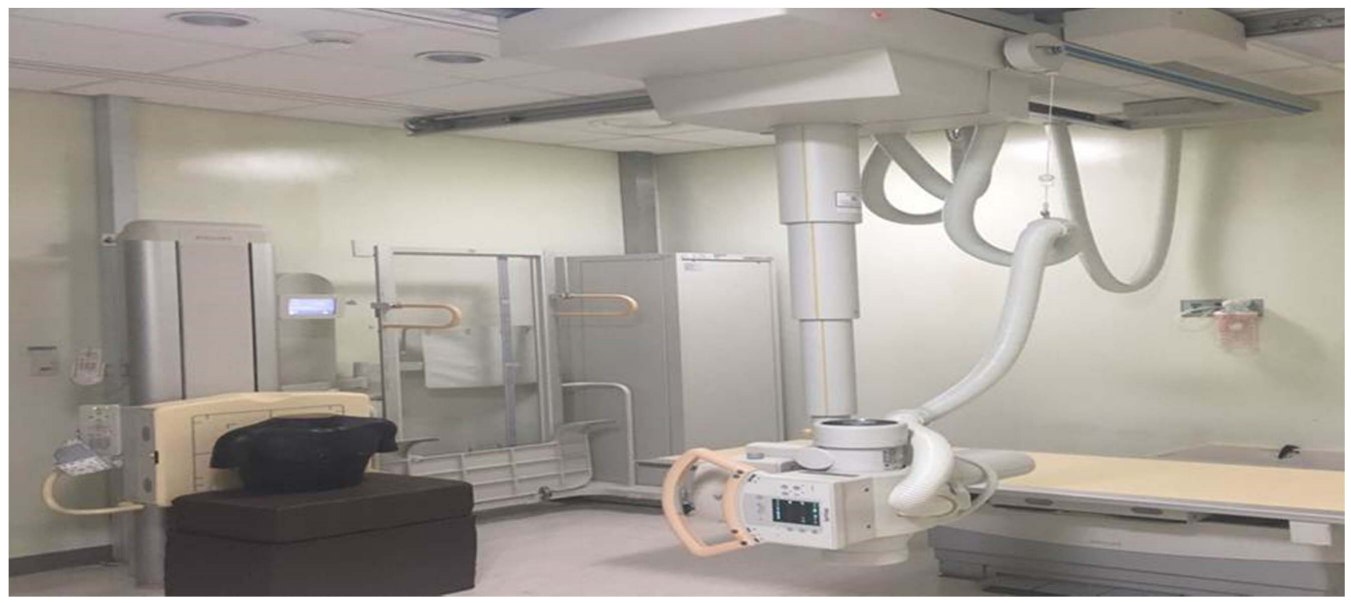

Fig. 2. (Color online) The photo of the experimental system with chest phantom. 
spatial frequencies $(\mathrm{lp} / \mathrm{mm})$. We implemented proposing algorithms by using the MATLAB (Version 8.3) programming language for the simulation study. Also, we acquired the chest image by using a general X-ray imaging system. Figure 2 shows the description of the X-ray system and the chest phantom used in this study. The Xray system is mainly consisted of an X-ray generator (120 $\mathrm{kVp}$ and $3 \mathrm{mAs}$ ) and a CMOS-type flat-panel detector having a pixel size of $200 \mu \mathrm{m}$. Also, the chest phantom consisted of anterior-posterior (AP) thickness of approximately $20 \mathrm{~cm}$.

\subsection{Evaluation of image quality}

NPS was measured for resolution and chest phantoms in X-ray imaging system. NPS represents the observational noise amplitude and texture at acquired image by homogeneous radiation which has specific photon flux and quality. Initial NPS (called by one dimensional NPS) is calculated by the slit synthesis method. However; because of its long processing time, the initial NPS method is continuously being decreased. In order to improve the processing time, Dainty and Shaw expressed two dimensional NPS on the following [15]:

$$
\begin{aligned}
& N P S(u, v)=\lim _{X, Y \rightarrow \infty} \frac{1}{2 X \cdot 2 Y} \\
& \left\langle\left|\int_{-X}^{X} \int_{-Y}^{Y}[I(x, y)-\bar{I}] e^{-\pi i(u x+u y)} d x d y\right|^{2}\right\rangle
\end{aligned}
$$

where $I$ is the image intensity, $\bar{I}$ is the average of the background intensity, and $\langle>$ accounts for the whole average. Additionally, the derived NPS should be considered, based on discrete frequency components of sample images in digital X-ray imaging system. Also, the first image of each X-ray system acquisition was only used to minimize the effect of lag on NPS measurement. Therefore, two-dimensional NPS mathematical expression is calculated as follows:

$$
\begin{array}{rl}
N & P\left(u_{n}, v_{k}\right)=\lim _{N_{x}, N_{y} \rightarrow \infty}\left(N_{x} N_{y} \Delta x \Delta y\right)\left\langle\left|F T_{n k} I(x, y)-S(x, y)\right|^{2}\right\rangle \\
= & \lim _{N_{x}, N_{y} \rightarrow \infty} \lim _{M \rightarrow \infty} \frac{\left(N_{x} N_{y} \Delta x \Delta y\right)}{M} \sum_{m=1}^{M}\left|F T_{n k} I(x, y)-S(x, y)\right|^{2} \\
= & \lim _{N_{x}, N_{y}, M \rightarrow \infty} \frac{\Delta x \Delta y}{M \cdot N_{x} N_{y}} \sum_{m=1}^{M} \\
& \left\langle\left|\sum_{i=1}^{N_{x}} \sum_{j=1}^{N_{y}}\left(I\left(x_{i}, y_{j}\right)-S\left(x_{i}, y_{j}\right)\right) \exp \left(-2 \pi i\left(u_{n} x_{i}+v_{k} y_{i}\right)\right)\right|^{2}\right\rangle
\end{array}
$$

where $\mathrm{S}$ is the average of background intensity, $N_{x} N_{y}, \Delta x$, $\Delta y$ account for pixel number of $\mathrm{X}, \mathrm{Y}$ axis and pixel size in $\mathrm{X}, \mathrm{Y}$ axis, respectively. Finally, we used NNPS to improve accuracy of NPS in this study. The NNPS is calculated as follows:

$$
N P S_{\text {normalized }}(u, v)=\frac{N P S(u, v)}{(\text { large area signal })^{2}}
$$

To evaluate the image quality between contrast and noise, we estimated CNR. With the CNR value, we indicated the difference of contrast in acquired images which are applied by each noise reduction technique. Y. N. Choi et al. expressed the CNR as follows [16]:

$$
\mathrm{CNR}=\frac{\left|S_{b}-S_{c}\right|}{\sqrt{N_{b}^{2}+N_{c}^{2}}}
$$

where $S_{c}$ and $S_{b}$ are the average value of the contrast components and the mean value of the background, respectively and $N_{c}$ and $N_{b}$ account for the contrast components and noise measured in the background, respectively.

\section{Results and Discussion}

Figure 3 shows the bar phantom image for the various noise reduction techniques in the simulation. We quantitative evaluated NNPS and CNR using a ROI A, B and $\mathrm{C}$ in the acquired images. In order to accurately evaluate image quality, we established same size of ROIs.

Figures 4 and 5 show the results for NNPS and the CNR as function of noise reduction techniques with bar phantom images, respectively. In the noisy bar phantom image, the NNPS result was distributed about regularly

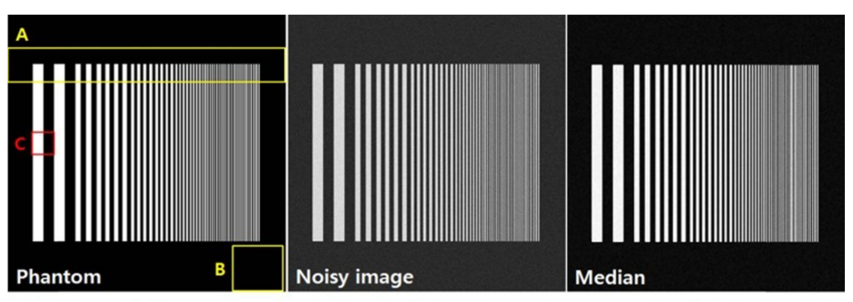

(a)

(b)

(c)

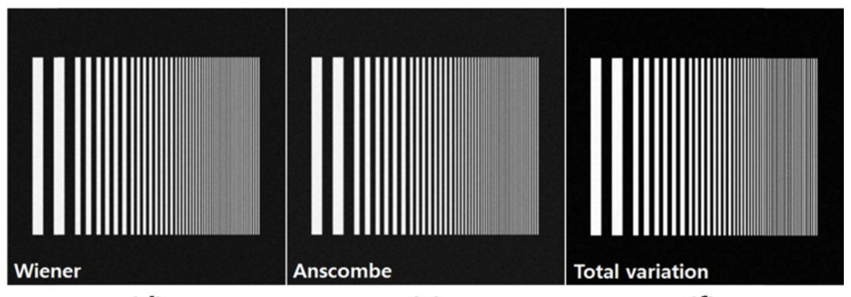

(d)

(e)

(f)

Fig. 3. (Color online) The simulated bar phantom images for (a) original, (b) noisy, (c) median filter, (d) Wiener filter, (e) Anscombe noise reduction technique and (f) TV noise reduction technique. 


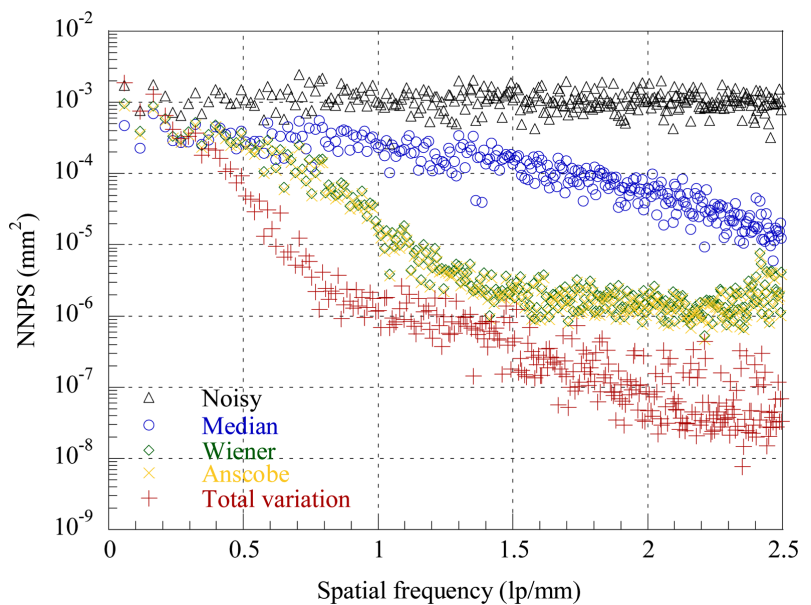

Fig. 4. (Color online) The results for the NNPS for noisy, median filter, Wiener filter, Anscombe noise reduction technique and TV noise reduction technique. When we used TV noise reduction technique, the lowest value was acquired in all frequency.

$10^{-3} \mathrm{~mm}$ in the range from 0 to $2.5 \mathrm{lp} / \mathrm{mm}$ spatial frequency. When we used the noise reduction techniques, the NNPS result is gradually decreased compared to the noisy

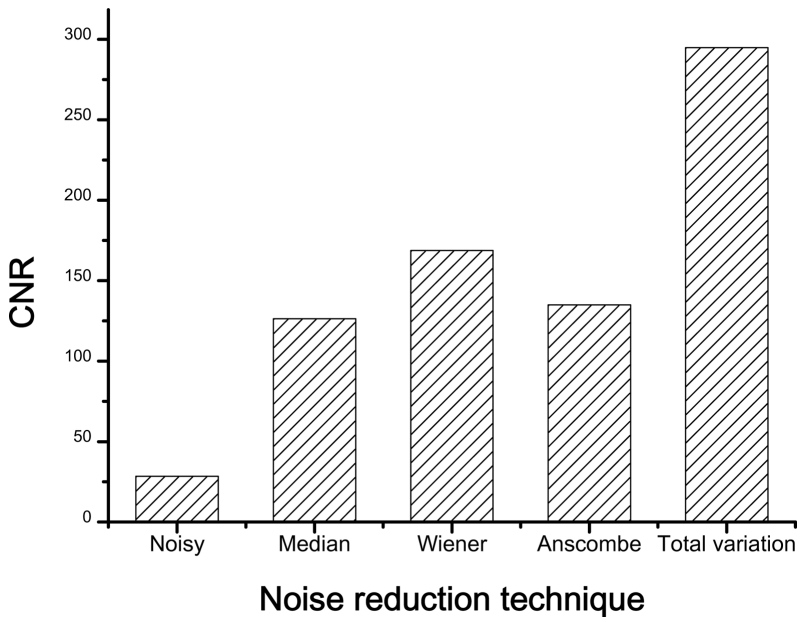

Fig. 5. The results for the CNR for noisy, median filter, Wiener filter, Anscombe noise reduction technique and TV noise reduction technique.

bar phantom image according to the increase of spatial frequency. Using Wiener filter and Anscombe noise reduction techniques, we acquired the NNPS result, which is the constant distribution with $10^{-6} \mathrm{~mm}^{2}$ when we used

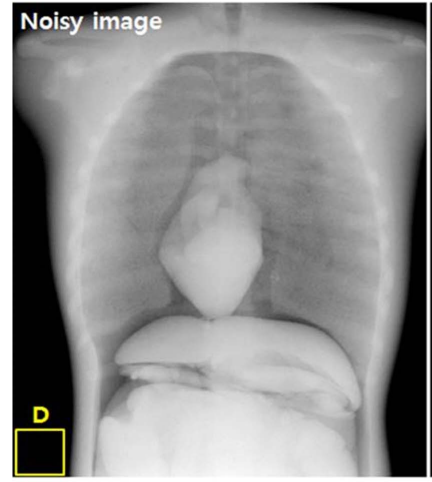

(a)

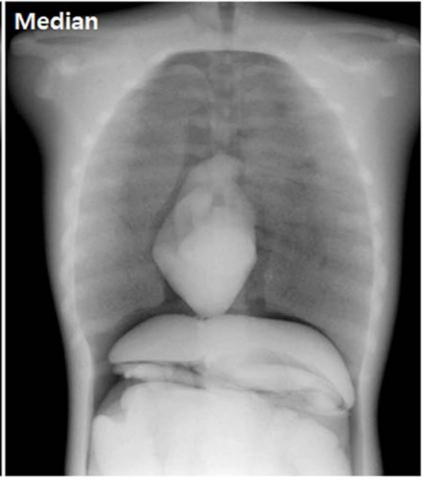

(b)

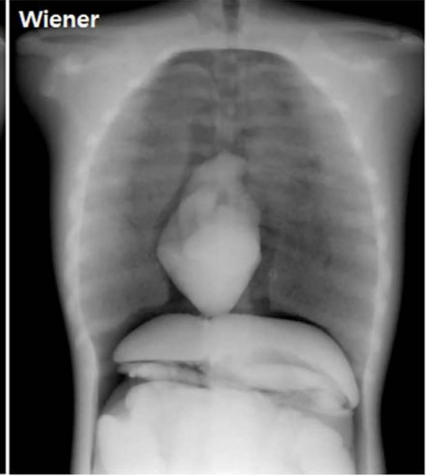

(c)

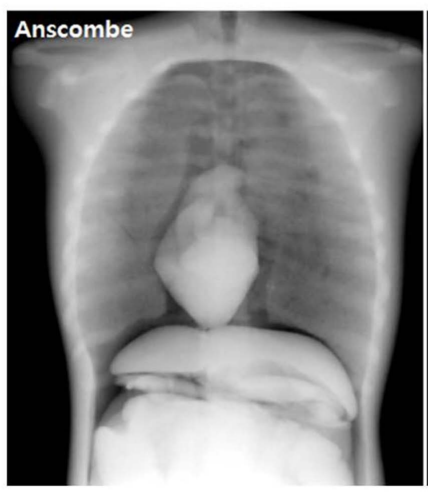

(d)

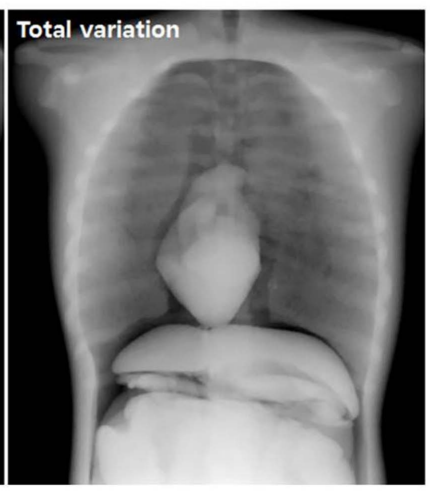

(e)

Fig. 6. (Color online) The acquired chest phantom images for (a) noisy, (b) median filter, (c) Wiener filter, (d) Anscombe noise reduction technique and (e) TV noise reduction technique. 


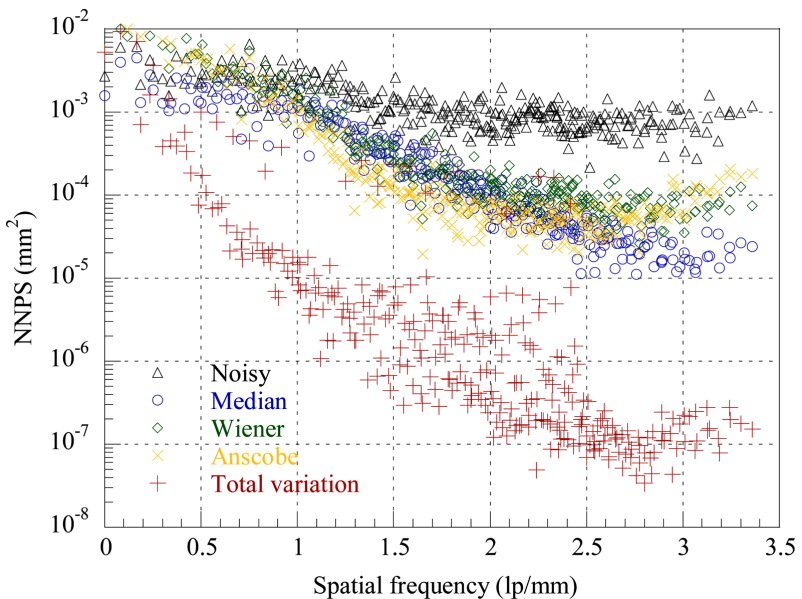

Fig. 7. (Color online) The results for the NNPS using ROI D for noisy, median filter, Wiener filter, Anscombe reduction technique and TV reduction technique.

over $1.5 \mathrm{lp} / \mathrm{mm}$ spatial frequency ranges. Especially, the NNPS results were acquired with approximately $10^{-8}$ $\mathrm{mm}^{2}$ using TV proposed noise reduction technique. The noise distribution for TV noise reduction technique was approximately 1.5 and 2 times lower than Wiener filter or Anscombe noise reduction technique and median filter, respectively.

The measured CNR value for the noisy bar phantom image, median, Wiener filters, Anscombe and TV noise reduction techniques were about 28.4, 126.4, 168.8, 134.9 and 294.8, respectively. In this result, TV noise reduction technique was 2.3, 1.7 and 2.2 times higher than median, Wiener filters and Anscombe noise reduction technique, respectively.

Figure 6 shows the chest phantom image for the various noise reduction techniques in the real experiment. Figure 7 shows the results for NNPS using ROI D as function of noise reduction techniques with chest phantom images.

In chest phantom images, the NNPS result was distributed about regularly $10^{-3} \mathrm{~mm}$ in the range from 0 to $3.5 \mathrm{lp} / \mathrm{mm}$ spatial frequency. According to the results, three different techniques-median filter, Wiener filter and Anscombe noise reduction technique-showed a similar tendency. In the techniques listed above, we acquired constant distribution with $10^{-6} \mathrm{~mm}^{2}$ when we used over $1.5 \mathrm{lp} / \mathrm{mm}$ spatial frequency ranges. Especially, the NNPS results were acquired with approximately $10^{-7} \mathrm{~mm}^{2}$ using TV proposed noise reduction technique. The noise distribution for TV noise reduction technique was approximately 2 times lower than Wiener filter, Anscombe noise reduction technique and median filter.

According to the results, we were able to reduce the noise performance with the filters and the noise reduction techniques that are used in quantitative evaluation. Wiener filter is often applied in the frequency domain with the Discrete Fourier Transform (DFT) to obtain degraded images. In Anscombe noise reduction technique, the noise can be treated as additive Gaussian. Both Wiener filter and Anscombe noise reduction technique are the degraded by image blurring. These are unable to reconstructed frequency components that have been degraded by noise components. Also, the image using median filter caused lower blurring and relative high edge preservation. However, TV noise reduction technique can be acquired the lowest noise component with preserved edge area.

\section{Conclusion}

The purpose of this study was to confirm effectiveness of TV technique for noise reduction in X-ray image. In this study, we evaluated and compared the image quality with various noise reduction techniques using NNPS and CNR. Through simulations and experiment, we were able to acquire the lowest NNPS in all frequency ranges and the highest $\mathrm{CNR}$ value in both simulation and experiment. In conclusion, our results successfully confirmed that proposed TV noise reduction technique is the efficient and practical technique and promising to application in the field of medical imaging.

\section{Acknowledgment}

This paper was supported by Eulji University in 2016.

\section{References}

[1] D. R. K. Brownrigg, Communications of the ACM 27, 807 (1984).

[2] T. Chen, K. K. Ma, and L. H Chen, IEEE Transactions on Image Processing 8, 1834 (1999).

[3] A. Somkuwar and S. Bhargava, $2^{\text {nd }}$ International Conference on Mechanical, Electronics and Mechatronics Engineering (2013) pp. 115-119.

[4] M. Makitalo and A. Foi, IEEE International Conference on Acoustics Speech and Signal Processing (2012) pp. 1081-1084.

[5] M. Makitalo and A. Foi, IEEE Transaction on Image Processing 20, 2697 (2011).

[6] J. Chen, J. Benesty, Y. Huang, and S. Doclo, IEEE Transaction on Audio, Speech and Language Processing 14, 1218 (2006).

[7] Q. Chen, P. Montesinos, Q. S. Sun, P. A. Heng, and D. S. Xia, Image and Vision Computing 28, 298 (2010).

[8] A. Buades, B. Coll, and J. M. Morel 4, 490 (2005).

[9] C. R. Vogel and M. E. Oman, SIAM Journal on Scientific 
Computing 17, 227 (1996).

[10] C. Tomasi and R. Manduchi, IEEE International Conference on Computer Vision (1998) pp. 839-846.

[11] W. Dong, L. Zhang, G. Shi, and X. Li, IEEE Transactions on Image Processing 22, 1620 (2013).

[12] K. Dabov, A. Foi, V. Katkovnik, and K. Egiazarian, IEEE Transactions on Image Processing 16, 2080 (2007).

[13] V. N. P. Raj, IEEE Transaction on Recent Advances in Intelligent Computational Systems (RAICS) (2011) pp.
483-488.

[14] L. Rudin, S. Osher, and E. Fatemi, Physica D 60, 259 (1992).

[15] J. T. Dobbins III, E. Samei, N. T. Ranger, and Y. Chen, Medical Physics 33, 1466 (2006).

[16] Y. N. Choi, S. W. Lee, H. M. Cho, H. J. Ryu, Y. J. Lee, and H. J. Kim, Journal of the Korean Physical Society 59, 3114 (2011). 